\title{
The Process of Wallerian Degeneration of Myelinated Nerve Fibers in the Pyramidal Tract of the Rhesus Monkey
}

\author{
By
Yoshiro INOUE, Toshio TERASHIMA, Yozo NISHIMURA and KAZUYO SHIMAI \\ Department of Anatomy, School of Medicine, Keio University, \\ Shinanomachi, Shinjuku-ku, Tokyo 160, Japan \\ -Received for Publication, January 20, 1978-
}

\begin{abstract}
Key Words: Wallerian degeneration, Central nervous system, Phagocytes, Pyramidal tract.
\end{abstract}

\begin{abstract}
Summary. 1. The process of Wallerian degeneration was investigated in the pyramidal tract of rhesus monkeys which had experimental lesions in the brain and had survived for a variety of periods, from 3 to 787 days, af ter physiological and neurological studies of the pyramidal tract lesions. 2. The nervous tissue samples were embedded in Epon 812 and observed by electron microscopy of ultra-thin sections and by light microscopy of semi-thin sections. In this way, it was shown that both normal and degenerated nerve fibers could be clearly identified, even by light microscopy. 3. The thick fibers of the pyramidal tract were attacked and engulfed by phagocytes at an early stage of 7 days after operation in such a manner that the phagocytes invaded the myelin tubes via the nodes of Ranvier, and engulfed the fragmented axons and myelin lamellae which had been peeled off from the paranodal regions. However, these phagocytes soon disappeared from the degenerated areas. Phagocytes then reappeared rapidly among the nerve fibers about 9 weeks later, and intensively phagocytosed the degenerated myelin sheaths which had not been consumed at the early stage, converting them into lipid droplets. These phagocytes may be more reasonably considered as reactive microglia, or if not so, exogenous macrophages. 4. The thin fibers were degenerated slowly and disappeared probably by phagocytosis and by an autolytic process.
\end{abstract}

There has been much controversy over the process of Wallerian degeneration in the central nervous system (Kusama and Nakazawa, 1975). Morphological studies on this problem have been less numerous than those on the peripheral nervous system and the kinds of the experimental animal species and nerve tracts used have varied widely. In the present study, since an opportunity arose to autopsy the brain and spinal cord of rhesus monkeys whose brain had been injured for clinical and physiological studies of pyramidal tract lesions, and in which there was a variety of survival times, we attempted to investigate the process of Wallerian de- 
generation of the pyramidal tract by electron microscopy of ultra-thin sections and light microscopy of semi-thin sections. Moreover, after identifying the degenerated and normal nerve fibers by electron microscopy, we attempted to examine the relative state of the degenerated nerve tract using semi-thin sections at various levels of the brain and spinal cord, and to discuss the possibility of the quantitative study on the normal and degenerated nerve fibers remaining in the degenerated nerve tract at both the light and electron microscopic levels.

\section{Materials and Methods}

1. The rhesus monkeys used in this study had been injured as follows.

Case I: the left motor cortex of the upper limb region had been ablated 7 days previously, and the right motor cortex of the upper limb region had also been ablated 3 days previously before the monkey was sacrificed. For convenience, the left medullary pyramid examined is thus termed Case-I-L, and the right one Case-I-R.

Case $I I$ : this monkey had been operated on three times. Left pedunculotomy had been performed first 293 days previously, the right motor cortex of the upper limb region had next been albated 19 days previously, and right pedunculotomy had been performed 12 days previously before the monkey was sacrificed. The left medullary pyramid studied thus constitutes Case-II-L, and the right one CaseII-R.

Case III: left pedunculotomy had been performed almost completely 25 days previously.

Case IV: the left internal capsule had been destroyed stereotaxically 33 days previously.
Case $V$ : left pedunculotomy had been performed incompletely 43 days previously.

Case VI: the left internal capsule had been destroyed 76 days previously.

Case VII : the left internal capsule had been destroyed 117 days previously.

Case VIII : the left motor cortex of the lower limb region had been ablated twice, at 787 days and then at 652 days previously.

Case IX: the left motor cortex of the upper limb region had been ablated 761 days previously and left pedunculotomy had been performed 645 days previously.

As controls, the bilateral medullary pyramids of five rhesus monkeys in which only small parts of the cauda equina had been injured, were used.

2. The rhesus monkeys, weighing some 3 to $4 \mathrm{~kg}$, were sacrificed by perfusion fixation with about $700 \mathrm{ml}$ of a mixed solution of cold $4 \%$ paraformaldehyde and $0.5 \%$ glutaraldehyde in Millonig's solution with or without pretreatment by perfusion of physiological Ringer's solution to flush out the blood. The solution was injected via the abdominal aorta or femoral artery and was discharged via the vena cava inferior or femoral vein, respectively.

After the perfusion, which took about 20 minutes, the body of the rhesus monkey was left undisturbed in crushed ice for one hour. The brain and spinal cord were then removed. Transverse sections of the medullary pyramids and each level of the spinal cord were cut and the material was divided into right and left halves. These tissue blocks were immersed overnight in the same fixative as that used for the perfusion. They were postfixed with $2 \%$ osmic acid in Millonig's buffered solution for 2 hours, following washing with the same buffered solution. The tissue blocks were flatly embedded 
into Epon 812 resin in the usual way to facilitate transverse sectioning.

Each half of the pyramid and spinal cord was cut transversely into slices 0.75 $\mu \mathrm{m}$ to $1.5 \mu \mathrm{m}$ thick with a Porter-Blum JB-4 microtome. The tissue was stained with $0.5 \%$ toluidine blue in $1 \%$ borax solution or $1 \%$ paraphenylenediamine aqueous solution for light microscopy of the semi-thin sections. The sections were not mounted in any media for observation under the light microscope, using a No-cover lens as an objective lens.

Further ultra-thin sections were cut from appropriate areas selected by light microscopy. These ultra-thin sections were double-stained with $2 \%$ aqueous uranyl acetate solution and Sato's mixed lead solution and were observed under an electron microscope (Hitachi HU-12AS).

The process of Wallerian degeneration in the pyraminal tract was observed chiefly from the transverse sections of the medullary pyramids in the present study, and was then compared with that at each level of the spinal cord.

\section{Results}

1. Normal nerve fibers in the medullary pyramid as revealed by electron microscopy

In the medullary pyramid of the rhesus monkeys, approximately $95 \%$ of the myelinated nerve fibers were of less than $3 \mu \mathrm{m}$ in diameter and thick myelinated nerve fibers were predominantly few in number. In particular, fibers thicker than $7 \mu \mathrm{m}$ formed only from one to two per cent of the total (Inoue et al., 1976), which corresponded to 7,000 to 10,000 as an absolute number. This thickest type of fiber may possibly originate from Bezt cells in the motor cortex.

Electron microscopy of normal myelinated nerve fibers which had been sectioned transversely, clearly revealed neu- rofilaments, microtubules, mitochondria, etc. (Fig. 1). The neurofilaments were from 80 to $160 \AA$ in thickness, forming bundles consisting of 10 or more neurofilaments, which ran longitudinally along the length of the axon. In these bundles, each of the neurofilaments was arranged in parallel at an interval of from 40 to $60 \mathrm{~nm}$. The neurofilaments were linked together by bridges of amorphous, electron-dense material (Fig. 1).

The microtubules, approximately $240 \AA$ in diameter, ran longitudinally and were dispersed in the axon. As a rule, the axons of thick fibers tended to contain more neurofilaments, while the axons of thin fibers tended to be richer in microtubules. Thus, the distribution of microtubules was generally such that they were fewer when neurofilaments were abundant, but numerous when neurofilaments were less abundant.

The mitochondria had a dark matrix, showing an almost circular outline 200 to $320 \mathrm{~nm}$ in diameter in the transverse plane, and their cristae were tubular (Fig. 1).

The lamellae of the myelin sheaths were generally preserved in a regularly layered form, although dissociation or disorder of the myelin lamellae was occasionally observed in parts of certain myelin sheaths. Small empty spaces were also found between the axolemma and myelin sheaths in some myelinated nerve fibers. The cytoplasm of oligodendroglia was observed to form a thin layer in the outer loop on the surface of the myelin sheath and in the inner loop between the axolemma and myelin sheath.

2. Degeneration of myelinated nerve fibers in the medullary pyramid as revealed by electron microscopy

There were two types of degeneration processes in the myelinated nerve fibers. In one, the degeneration of thick nerve fibers of diameter more than about $3 \mu \mathrm{m}$, 
phagocytes participated at the early stage. In the other, chiefly the degeneration of thin nerve fibers, the changes in the fibers progressed slowly.

(i) The degeneration process of thick nerve fibers

At 3 days after operation (Case-I-R), myelinated nerve fibers with numerous filamentous structures (Fig. 2) or ones in which microtubules were unusually abundant, were found among the normal nerve fibers. These features were considered to represent early changes of degenerated nerve fibers. The interval between the filaments of degenerated axons became narrower, about 20 to $30 \mathrm{~nm}$ and the density of the filament population increased markedly compared to that of normal axons. These prolific filaments had clear contours, since there were few bridges of amorphous substance linking them together (Fig. 2). Bundles of these filaments ran rather irregularly along the length of the axons, since they tended to be arranged longitudinally and obliquely in the transverse sections. In the degenerated axons, which contained unusually abundant microtubules, there were very few neurofilaments. In the degenerated axoplasm, mitochondria occurred in greater numbers (Fig. 2), and their cristae appeared irregular in shape and obscure since the matrix became more electrondense. Tubular or saccular structures were found to be more abundant, especially just beneath the axolemma.

These changes at the earliest stage were followed by more dramatic events such as fragmentations of degenerated axons, shortening of the internodal segments of myelin sheaths of degenerated nerve fibers, and the appearance of phagocytes within myelin sheaths.

At 7 days after operation (Case-I-L), various unusual changes in the axoplasm and myelin sheaths were found in cross sections of the medullary pyramid as follows :

1. Collapsed and empty myelin sheaths in which the axon had disappeared (Fig. 3-a).

2. Myelin sheaths enclosing a shrunken axon with light and floccular or dark and finely-granulated axoplasm (Fig. 3-b).

3. Three concentric rings of myelin sheaths of approximately normal thickness, in which no axon (or only a vestige of a degenerated axon) remained in the narrow central space (Fig. 3-c).

4. Unusual myelin masses with a concentric arrangement such that the outer ring was very thick and its myelin lamellae too obscure to be identified, while the central ring was a approximately normal thickness for a myelin sheath and its lamellar structure relatively clearly discernible (Fig. 3-d).

5. Unusual myelin masses resembling those in 4 but with the arrangement of concentric myelin rings reversed so that the outer ring was of approximately normal thickness for a myelin sheath, while the central region consisted of a spherical myelin mass of large diameter whose lamellar structure was not clearly identifiable. The central space was absent or, if present, very narrow and sometimes filled with a small part of the degenerated axon.

Based on these findings, it was considered that the axons of degenerated nerve fibers might be fragmented and discontinuous along their whole length within the myelin sheaths, and in addition, that the myelin sheaths might be invaginated into their own myelin tube to give rise to shortening of their internodal segments. The unusual features of the myelin sheaths found in the cross sections could be explained by drawing a schema for the invaginating myelin tubes in which the findings listed under items 1 to 5 above probably corresponded to the features of the cross section though 
the regions $a^{\prime}$ to $e^{\prime}$ in the schema, respectively (Fig. A).

It should also be emphasized that at this early stage phagocytes took part in the degeneration process by engulfing fragments of degenerated axons and shortened myelin sheaths. That is to say, at 7 days after operation, phagocytes were frequently found invading collapsed myelin tubes, engulfing the degenerated axons with a light and floccular (Fig. 5) or dark and finely-granulated matrix (Fig. 4). The cytoplasm of the phagocytes enclosed the degenerated axon, forming a small pile of thin cytoplasmic sheets around the axon fragment (Fig. 5). The nucleus possessed abundant chromatin associated with the nuclear envelope, and there was a clear-cut border against the nucleolymph (Fig. 4). The nucleolus also contained associated chromatin. The rough-surfaced endoplasmic reticulum was found to be dispersed, but the cisternae were sometimes piled into a few layers which contained electron-dense material and were especially bulged in their peripheral parts. The enlarged portions were apparently released from the cisternae of the endoplasmic reticulum to form vacuoles with electron-dense material, estimated at about 0.3 to $0.5 \mu \mathrm{m}$ in diameter (Figs. 4 and 5). Occasionally, a small amount of ribosomes was found attached to the surface of these vacuoles.

Vesicles or short rod-like bodies which were more electron-dense but smaller in size than the above vacuoles, occurred around the rough-surfaced endoplasmic reticulum (Fig. 4). They were noticeably abundant in the cytoplasmic tongue which had enclosed the degenerated axon fragment or had invaded the space between the lamellae of myelin sheaths (Fig. 6).

The Golgi apparatus was rarely observed, but free ribosomes were frequently present. Tubular cristae of mitochondria were relatively well developed.
On the other hand, fragments of degenerated axons, not being phagocytosed at the early stage, may disappear by an autolytic process. At 25 days after operation (Case III), axons with dark and finelygranulated axoplasm were still found frequently in spite of a remarkable disappearance of phagocytes from the inside of the myelin sheaths, while in the later stages at 33 and 43 days after operation (Case IV and V, respectively) the degeneration of the axons had so advanced that they were swollen and had disintegrated into necrotic debris within the myelin tubes. Thus, degenerated axons probably disappear as a result of autolytic breakdown as well as by phagocytic engulfment.

Those degenerated myelin sheaths whose internodal segments has been shortened by invagination associated with fragmentation of the axons, were also partially engulfed by phagocytes which had occupied the lumen of the myelin tubes. Occasionally the axon fragment was simultaneously phagocytosed (Fig. 6). At 7 days after operation (Case-I-L), the phagocytes within the myelin sheaths were frequently found to have extended their cytoplasmic processes between the myelin lamellae. Since paranodal loops of the node of Ranvier were sometimes observed at the entry point of invasion, the phagocytes probably peeled off myelin lamellae from the paranodal region to engulf them (Fig. 6).

At 19 days after the first operation (i.e. 12 days after the second operation) (CaseII-R), phagocytes engulfing myelin were found within thin remnants of myelin lamellae which had been peeled off from the inside or within the myelin tubes of approximately normal thickness. The cytoplasmic structure of the phagocytes at this stage was somewhat different from that of the phagocytes observed within the myelin sheaths at 7 days after opera- 
tion (Fig. 7). That is to say, in the phagocytes engulfing myelin fragments, vacuoles of about 300 to $500 \mathrm{~nm}$ in diameter were found to have increased in number, although the relatively electron-dense content became partially electron-lucent in some of the vacuoles. Numerous empty vacuoles of various sizes were also present. Golgi complex with well-developed cisternae and vacuoles was clearly observed, while rough-surfaced endoplasmic reticulum with smaller but distended cisternae was dispersed. In particular, filamentous structure of approximately $70 \AA$ in diameter came to be found. The phagocytes commonly engulfed more than one myelin fragment, and these were found to be membrane-bounded. In some of the myelin fragments, the normal laminated structure of myelin sheaths was well preserved, but others had disintegrated into irregular, vesiculated and lacunal masses (Fig. 7).

Since in other phagocytes engulfing great amounts of myelin fragment and containing numerous empty vacucles, the nucleus had a pyknotic appearance and the detailed structure of the cytoplasm became obscure, it was considered that most of the phagocytes within the myelin sheaths may die and undergo autolytic breakdown after the completion of phagocytosis (Fig. 8). From this stage on, the necrotic tissue debris was commonly observed to occur in the myelin remnants of thin walls.

At 25 days after operation (Case III), phagocytes were rarely observed within the myelin sheaths in spite of the fact that most nerve fibers were undergoing degeneration, although necrotic tissue was frequently observed there. Occasionally phagocytes engulfing myelin fragments were seen among the nerve fibers, and the cytoplasm of astrocytes showed a remarkable increase in volume. In the Virchow-Robin space around the blood vessels, on the other hand, phagocytes engulfing myelin fragments sometimes occurred at this stage (Fig. 9). The rapid decrease in the phagocytes which had earlier appeared within the myelin sheaths, might thus be due to their autolytic breakdown following overconsumption of myelin and axon fragments, and to their migration to blood vessels.

At 3 weeks after operation, most of the degenerated myelin sheaths remained as concentrically layered myelin masses which had probably formed by invagination of myelin tubes, collapsed myelin tubes with an almost normal thickness of lamellae, and thin myelin remnants which phagocytes could no longer have engulfed at the earlier stage. The outer layer of the concentric masses of myelin sometimes became thinner, probably due to peeling off of lamellae by phagocytes from the inside.

At 25 days and 33 days after operation (Cases III and IV, respectively), the processes and some of astrocytes became so hypertrophic and expanded in volume among the nerve fibers that some of the processes had invaded into the myelin tubes (Fig. 10). However, phagocytes were never observed to engulf a large amount of myelin masses, only small pieces, if any, although the cytoplasm of the phagocytes was sometimes found to lie in contact with degenerated myelin masses. Astrocytes did not take part in the phagocytosis during the entire degeneration process.

The degenerated myelin remnants left from phagocytosis may undergo an autolytic process. At 25 days, 33 days and 43 days after operation, some of the concentrically arranged myelin masses, and collapsed myelin sheaths, gradually gave rise to dissociated myelin lamellae and became rich in vacuoles and lacunae. They might then be said to approach an appearance in which only the outer wall 
of very thin myelin lamellae remained and enclosed the necrotic debris from the disintegrated myelin within it. At 117 days after operation (Case VIII) the degenerated nerve fibers were distinctly fewer in number, although much degenerated myelin remained which gave the appearance of distended myelin tubes with extremely thin walls as mentioned above. Also large myelin masses of the kind found at the earlier stages were still present, even though dissociation of the myelin lamellae had occurred.

At 293 days after operation (Case-III-L), there was more or less some degenerated nerve myelin, whereas at 761 days and 787 days after operation (Case IX and Case VIII, respectively), the degenerated nerve fibers and disappeared completely.

As mentioned above, the phagocytes which had appeared at the early stage of the degeneration process to engulf part of thick degenerated axons and myelin sheaths, were found to disappear mostly from the degenerating nerve tract within about 3 to 4 weeks after operation. However, at more than about 9 weeks after operation, phagocytes showed a tendency to reappear rapidly among the nerve fiber (Fig. 11). This new occurrence of phagocytes could depend to a large extent on the stage of the degenerating process, even though the number of phagocytes appearing could be related to the amount of degenerated nerve fibers. For example, comparing the situation at 43 days after operation (Case V) with that at 76 days after operation (Case VI), the degenerated nerve fibers in the pyramid of the latter animal were fewer than those in the former, but the number of phagocytes containing lipid droplets and myelin fragments in the pyramid of the latter was about twice that in the former case. These phagocytes among the nerve fibers revealed the features similar to microglia observed previously in the rhesus monkey optic nerve (Inoue et al., 1976), possessing relatively well-developed Golgi complex and small rod-like structures containing electron-dense material, a small amount of dispersed rough-surfaced endoplasmic reticulum with short cisternae, and a few ribosome. However, as the phagocytes came to contain abundant lipid droplets of low electron density, probably as a result of the digestion of engulfed myelin fragments, so the cytoplasm was pushed away and the external shape of the nuclei became changed. Some of the lipid droplets were found to be bordered with unit membrane from the cytoplasm, but the others were not clearly observed to be limited. The lipid droplets were estimated to be approximately $1.5 \mu \mathrm{m}$ in diameter and were often fused (Fig. 12). Between these lipid droplets, laminated bodies were also found in which three-layered laminae like a limiting membrane of about $130 \AA$ in thickness piled up at intervals of $40 \AA$ in width at the narrowest to form needle-shaped or angular-shaped bodies as a whole. Large laminated bodies were rarely observed until 76 days after operation (Case VI), although they and lipid droplets still occurred in phagocytes at 293 days after operation (Case-II-L). At 761 days and 787 days after operation (Cases IX and VIII, respectively), there were no cells containing lipid droplets.

(ii) The degeneration process of thin nerve fibers

At 7 days after operation (Case-I-L), thin nerve fibers of less than about $3 \mu \mathrm{m}$ in diameter came to possess electron-dense axons but relatively well-preserved myelin sheaths. Some degenerated myelin sheaths formed onion-like myelin masses, probably as a result of invagination of their own myelin tubes. However, since collapsed myelin sheaths were not frequently found, it was considered that such fragmentation of axons and shortening of the internodal 
segment of myelin sheaths as was frequently seen in the case of thick nerve fibers, may occur only with difficulty in thin nerve fibers. Such degenerated nerve fibers were uncommonly observed to be engulfed by phagocytes among the nerve fibers at 3 weeks after operation, while most of the degenerated fibers were enclosed by hypertrophied cytoplasmic processes of astrocytes and some were in direct contact with the phagocytes. At 117 days after operation (Case VII), numerous thin degenerated nerve fibers were still observed which did not show the remarkable changes in features occurring at the earlier stages, although the total number of nerve fibers had decreased (Fig. 12). At this stage there were numerous phagocytes containing abundant lipid droplets, and, moreover, these phagocytes were found to be simultatneously engulfing small myelin fragments. At 293 days after operation, degenerated nerve fibers were generally rare, except that the small onion-like myelin masses did remain. At 761 days and 787 days after operation, all degenerated nerve fibers had disappeared from the affected nerve tract. Thus, the thin degenerated nerve fibers were considered not to disappear at once, but to be lost by gradual phagocytosis and an autolytic process over a prolonged period.

\section{Light microscopy of semi-thin sections}

The above findings obtained by electron microscopy were also adequately identified by light microscopy using semi-thin sections prepared from the same tissue blocks embedded in Epon 812 resin as those used for the ultra-fine structural studies.

Normal nerve fibers possessed a light axoplasm enclosed by a blackly stained myelin sheath, and mitochondria were clearly observed within the axons as granular structures. The artificial spaces between the myelin lamellae or between the myelin sheath and axon could be clearly distinguished from the axoplasm (Fig. 13).

At 3 days after operation (Case-I-R), even though an increase in filamentous structures within the axons, which might represent the earliest changes of degeneration, was observed by light microscopy as a darkening of the axoplasm in typical cases, it was considered that all degenerated nerve fibers could not be identified (Fig. 13).

At 7 days after operation (Case-I-L), however, as in the case of electron microscopy, collapsed myelin rings or spherical myelin bodies which may have resulted from shortening of the internodal segments of myelin sheaths due to invagination in association with fragmentation of axons, were clearly observed (Fig. 14). Phagocytes occurred within the myelin sheaths and were seen peeling off myelin lamellae from the inside of myelin sheaths (Fig. 14). Blackening of axons of thin nerve fibers or the formation of myelin bodies was adequately identified under the light microscope (Fig. 14).

Following these earlier changes, it was clearly identified by light microscopy that phagocytes were engulfing myelin sheaths, myelin masses among the nerve fibers had broken down into a vesiculated and lacunal form probably as a result of an autolytic process, and thin myelin laminae remained with or without necrotic tissue debris within their distended lumen. Phagocytes in Case-II-R engulfed myelin fragments which were observed as numerous blackened inclusions. Their nuclei sometimes became pyknotic and their cytoplasm also became vacuolated or lacunal probably leading to the necrotic process (Fig. 15).

At a later stage, phagocytes containing numerous vacuoles (probably of the lipid droplets identified by electron microscopy) were demonstrated among the nerve fibers 
(Fig. 16).

Thus, it was considered that the degenerated nerve fibers in the medullary pyramids could be satisfactorily identified on an individual basis, and were distinguishable from the corresponding normal nerve fibers, in so far as they occurred in transverse sections.

4. The degeneration process at various levels of the spinal cord

The degeneration process described above represents that at the level of the medullary pyramid following primary lesions of cranial constituents of the pyramidal tract system. At lower level of this tract, i.e. at various levels of the spinal cord, the process and rate of degeneration were found to be almost as advanced as in the pyramid. For example, at 7 days after operation (Case-IL) in the lumber of the spinal cord where degenerated fibers had decreased in number, phagocytes also occurred within myelin sheaths in the region of the lateral corticospinal tract (Fig. 17), and at 117 days after operation, phagocytes containing numerous lipid droplets were found at the lumber level of the spinal cord, as in the pyramid of each respective case (Fig. 18).

It appeared reasonable therefore to consider that the amount of degenerated nerve fibers, which varied in the pyramid and spinal cord of each case used in the present study, might not influence on movement of phagocytes, and that distance from the lesions might not influence on the observed degeneration process.

\section{Discussion}

1. The process of Wallerian degeneration in the central nervous system

It has been reported that in the central nervous system Wallerian degeneration of thick nerve fibers advanced more rapidly than that of thin ones, although the reasons have not been rigorously discussed. In the pyramidal tract of the rhesus monkey, a difference in manner of disruption and disappearance between degenerated thick and thin fibers was also observed. At the early stage (CaseI-L), the axons of thick fibers, which were estimated to be more than about $3 \mu \mathrm{m}$ in diameter (in particular including fibers more than $7 \mu \mathrm{m}$ in diameter, probably of Bet $z$ cell origin), fragmented and their myelin sheaths were invaginated into their own lumens, resulting in shortening of the internodal segments and opening of the nodes of Ranvier. Thus, in the case of thick nerve fibers, physical morphological changes occurred in the myelin sheaths at the early stage, and further, phagocytes entered the myelin sheaths from the opened nodes of Ranvier to engulf the fragments of degenerated axon. These phagocytes within myelin sheaths simultaneously extended their cytoplasmic processes between the myelin lamellae, and peeled them off from the inside and engulfed them in great amounts. The thin nerve fibers, on the other hand, did not undergo remarkable changes except for darkening of the axoplasm and the formation of spherical myelin bodies at the early stage, and they were not significantly attacked and engulfed by phagocytes. It was considered that such a difference between thick and thin nerve fibers as regards their degeneration could be due to a variance in the tissue reactions to the newly appearing foreign bodies, of which the large foreign bodies arising from the degeneration of thick nerve fibers perhaps became the target of phagocytosis at the early stage.

Lampert (1969) observed that during Wallerian degeneration of rat posterior funiculus, which is a long projecting tract like the pyramidal tract, phagocytes invaded myelin lamellae from the outside 
via the nodes of Ranvier, the gaps created by focal myelinolysis or the slits beneath the outer loop of myelin sheaths, in order to strip them off and eugulf them. However, as described by Lampert et al. (Lambert, 1969 ; Lampert and Cressman, 1966), it was at a relatively late stage during the degeneration process that phagocytes appeared in order to consume the affected myelin sheaths, except in the primarily injured parts or peritraumatic region. There have been few reports of phagocytes appearing as early as those in the present study to disrupt the myelin sheaths. Concerning the occurrence of phagocytes within the myelin tubes during Wallerian degeneration, there are only the report of Cook (1974), who observed phagocytes consuming axoplasmic debris in degenerated optic nerve of the cat and monkey, but not the myelin lamellae, and that of Daniel and Strich (1969), who observed cells with a pyknotic nucleus within degenerated myelin sheaths at the early stage under a light microscope, and assumed that they might be referred to the so-called axophagia or myeloclasts of Jakob (1913).

Comparison of Wallerian degeneration of the pyramidal tract of rhesus monkeys with that of the optic nerve of the same animal (Cook and Wisniewski, 1973 ; Cook, 1974), reveals differences in the respective processes. During degeneration of the optic nerve, axon debris was sometimes found to be engulfed by phagocytes within the myelin sheaths at the early stage, as in the case of the degenerated pyramidal tract, but the myelin sheaths were never found to be engulfed until about 35 days postenucleation. On the other hand, in the pyramidal tract, the phagocytes invading the myelin tubes engulfed the myelin lamellae from the inside as early as at 7 days after operation, and further they reappeared rapidly at about 9 weeks after operation to consume and digest the myelin remnants and other degenerated nerve fibers which had not been engulfed at the early stage.

The phagocytes in degenerated optic nerve were identified as oligodendroglia (Cook and Wisniewski, 1973; Cook, 1974; Cook et al., 1974). In the present study, however, two types of the phagocytes, one occurring at the early stage within the myelin sheaths and the other at the later stage among the nerve fibers, were clearly distinguishable from typical oligodendroglia and astrocytes, which existed simultaneously in the examined pyrmidal tract. Especially the type of the later stage could not be distinctly identified from resting microglia occurring among the nerve fibers in the uniujured medullary pyramid. The phagocytic cells in the pyramidal tract may thus be more reasonably considered as reactive microglia, or if not so, exogenous macrophages. The myelinated nerve fibers constituting the rhesus monkey optic nerve have been reported as distinctly less than $2 \mu \mathrm{m}$ in diameter (Inoue et al., 1976), whereas in the pyramidal tract, there are thick fibers from about $3 \mu \mathrm{m}$ to $10 \mu \mathrm{m}$ in diameter. Therefore, the reaction of the phagocytes in both case cannot be discussed on an equal basis due to the great difference in size of the degenerated myelin which is the object of removal as endogenous foreign bodies.

Data for the rat pyramidal tract (Dunkerly and Duncan, 1969) show that its nerve fibers are all less than $4 \mu \mathrm{m}$ in diameter, with $99 \%$ less than $3 \mu \mathrm{m}$. This clearly contrasts with the rhesus monkey pyramidal tract (Inoue et al., 1976) where, as described above, thicker fibers constitute about $5 \%$ of the total number of fibers, corresponding to half of the total number in the rat pyramidal tract. With such different nerve fiber constitutions, a variance in the reaction of phagocytes during Wallerian degeneration is to be 
expected. In the rat pyramidal tract, no phagocytosis of axon debris and myelin sheaths was observed untill 2 to 3 weeks after operation, and moreover, the phagocytic cells were identified as astrocytes or immature astrocytes. Explanation of this difference in the reaction of phagocytes in spite of the same type of tract, may thus depend on the different thicknesses of the nerve fibers constituting the tracts as well as the variety of animal species used.

The optic nerve has often been used for investigations of Wallerian degeneration since its nerve fiber constitution and glioarchitecture resemble those of the central nervous system. However, as pointed out by Anderson and his coworkers (Anderson, 1969; Anderson and Hoyt, 1969; Anderson et al., 1967) and by Inoue et al. (1976a, b), the optic nerve fibers of the rhesus monkey and other vertebrates are separated into numerous bundles by connective tissue septa, in which all the blood vessels, including the blood capillaries, are embedded and surrounded by collagenous fibrils. Therefore, a distinct difference exists between the blood vessel architecture of the optic nerve and that of other parts of the central nervous system. In the optic nerve, endothelial cells of the blood capillaries are never in direct contact with the basal lamina existing just beneath the cytoplasm of the glial limitants of the neural tissue, whereas in the white matter of the central nervous system endothelial cells of the capillaries are intimately contiguous with the glial limitans, although the basal lamina lies between them. It is possible that such a variance in vascular architecture could influence the movement of phagocytes during degeneration, if they would be hematogenous in origin.

Comparing the Wallerian degeneration of different tracts in different animal species reported in the literature, differ- ences in the degeneration process, especially in the reaction of phagocytes, are seen. In the case of the rat, various origins have been given for phagocytes. Vaughn et al. (1970) described phagocytes as multipotential cells in the optic nerve, Fernando (1973) described them as astroblasts in the pyramidal tract, and Lampert and Cressman (1966) as hematogenous mononuclear cells, although undeniably as microglia, in the posterior funiculus of the rat. On the other hand, Dunkerley and Duncan (1969) have denied the occurrence of phagocytosis of degenerated myelin lamellae in the rat pyramidal tract.

Concerning the cat, there are reports on degeneration of the optic nerve (Cook and Wisniewski, 1973) and of the posterior funiculus of the spinal cord (Bignami and Ralston, 1969). In the former study, degenerated myelin sheaths were observed to be engulfed by the cytoplasmic processes of oligodendroglia 4 to 5 weeks after enucleation, and in the latter study they were observed to be phagocytosed by macrophages at about 60 days after operation.

Another matter of controversy concerns the origin of phagocytic cells during Wallerian degeneration of the central nervous system. Astrocytes, oligodendroglia and microglia under normal conditions have been considered as adequately identified by electron microscopy (Mori and Leblond, 1969a, b, 1970; Inoue et al., 1976a, b). However, in the degenerated tract the glial cells, including phagocytic cells, all of which belonged to the interstitial cells of the nervous tissue, have often proved difficult to identify due to the reactive changes in these cells against the appearance of degenerated nerve fibers. It has been considered therefore that application of the criteria of identification suitable under normal conditions to such reactive cells, including the phago- 
cytic cells, might only lead to confusion over the origin of the phagocytes. In addition to the difficulty of identification, the above-mentioned variance in animal species and tracts investigated probably serves to render the problem of the origin of phagocytes even more complex. Even quite recently, among cells which have been reported to phagocytose myelin debris, a great variety persists, such as microglia (Lampert and Cressman, 1966; Fernando, 1973), oligodendroglia (Cook and Wisnieski, 1973), astrocytes (Lampert and Cressman, 1966; Gonatas et al., 1964 ; Fernando, 1973), and exogenous macrophages (Bunge et al., 1960 ; Ressel, 1962). Thus, although in this study it was tentatively concluded that relative microglia or exogenous cells might be responsible for the observed phagocytosis of degenerated nerve fibers, detailed work on phagocytes in general must be undertaken in the future.

2. Reaction of the phagocytes in degenerated nerve tracts

During the degeneration of the pyramidal tract of rhesus monkeys reported in this study, phagocytes reacted diphasically. They were first found at 7 days after operation in the early stage, and engulfed degenerated axons and myelin sheaths. Most of them were then subject to self-destruction although some were transferred to the blood vessels, digesting the myelin debris into lipid droplets. Thus, the early phagocytes disappeared rapidly from the degenerated tract in 3 to 4 weeks. Daniel and Strich (1969) have observed cells with a pyknotic nucleus within the myelin sheaths of degenerated posterior funiculus of the baboon at the early stage, and have reported that they disappeared within about 2 weeks.

Subsequently, in the degenerated pyramidal tract of the rhesus monkey, phagocytes containing myelin debris and lipid droplets were occasionally observed among the nerve fibers. However, after 9 weeks, there was a rapid increase in phagocytes consuming myelin debris and containing lipid droplets among the nerve fibers. The phagocytes appearing at this second phase were not so self-destructive as those observed in the first phase. They contained numerous lipid droplets and also laminar bodies or laminar inclusions of the kind described by previous authors (Lampert and Cressman, 1966; Tani, 1965 ; Bignami and Ralston, 1969; Daniel and Strich, 1969; Lambert, 1969; Cook and Wisniewski, 1973; Cook et al., 1974; Vaughn et al., 1970).

According to the previous papers listed above, it was at the later stage that the phagocytes began to engulf the myelin sheaths; that is, during Wallerian degeneration of cat posterior funiculus, phagocytosis began at 60 days after operation (Bignami and Ralston, 1969), in baboon posterior funiculus, at 31 to 52 days (Daniel and Strich, 1969), in cat optic nerve, at 56 days (Cook and Wisniewski, 1973), in rat posterior funiculus, at several weeks (Lampert and Cressman, 1966), and in rat pyramidal tract, at 2 to 3 weeks (Fernando, 1973). These results concerning phagocytic reactions might thus correspond to the second phase observed by us in the rhesus monkey.

In the later stage, the phagocytes may perhaps be considered to engulf myelin debris which has been surrounded by hypertrophic cytoplasm of astrocytes for prolonged periods so that its chemical constituents are different from those of the myelin masses at the early stage. Smith (1956) investigated Wallerian degeneration of human spinal cord by the Marchi method, and found that Marchi positive material appeared in the extracellular space for the first 10 weeks, but then tended to occur only intracellularly. McCaman and Robins (1959) studies the 
chemical and quantitative properties of Wallerian degenerated optic nerve in the rabbit, and found that at 100 days after operation the amount of lipid decreased rapidly and remarkably.

Thus, in the present study it is possible that the phagocytes occurring at the first phase might have been involved in the physical disruption of the large foreign bodies of the degenerated axons and myelin sheaths of thick nerve fibers for a short period. On the other hand, the phagocytes at the second phase might play a role in the chemical digestion of degenerated myelin remnants. Such a diphasic reaction of physical disruption followed by chemical digestion has often been indicated in the phagocytic process of Schwann cells during Wallerian degeneration of the peripheral nervous system (Lampert and Cressman, 1966 ; Kusama and Nakazawa, 1975).

3. Identification of normal and degenerated nerve fibers by light microscopy

Nauta's, Marchi's, Fink-Heimer's or Yamadori's method (1975) has been used to stain degenerated nerve fibers. However, the attainment of successful preparations with those method is strongly dependent on the survival time after operation, and only degenerated nerve fibers are visualized by the staining. It has thus been impossible to discuss rigorously the qualitative and quantitative deta of both the remaining normal fibers and degenerated nerve fibers in an injured tract by simple comparison with postoperative changes in behavior and, if any, functional recovery which can be continuously observed from the standpoint of clinical neurology.

In the present study, the morphological characteristics of normal and degenerated fibers in Wallerian degenerated pyramidal tract of rhesus monkeys after various postoperative survival times, were inves- tigated by electron microscopy and light microscopy of semi-thin sections. The results were compared, and it was found possible to distinguish degenerated fibers from normal ones not only by electron microscopy but also by light microscopy, even in the case of the thinnest fibers, provided that the degenerated myelin debris had not been engulfed and digested. The rate of degeneration was found to be almost equal between the various levels of the pyramidal tract from the medullary pyramid to the lower segments of the spinal cord, in which degenerated nerve fibers then decreased in number.

The method employed in the present study was thus considered suitable for making comparisons between actual brain injuries and functional or behavioral changes with special reference to changes in the constitution of the nerve fibers surviving after degeneration of a related nerve tract.

\section{Acknowledgements}

We wish to express our sincere thanks to Ms. Sumiko Hashimoto and Mr. Masaki Sekiguchi for their expert assistance. This study was supported by a special fund of The Science and Technology Agency of Japan for promoting multiministerial projects, 1973-1975, on "Studies on the Regeneration of Nervous Tissue".

\section{References}

1) Anderson, D. R.: Ultrastructure of human and monkey lamina cribrosa and optic nerve head. Arch. Ophthal. 82: 800-814, 1969.

2) Anderson, D. R. and Hoyt, W. F.: Ultrastructure of intraorbital portion of human and monkey optic nerve. Arch. Ophthal. 82 : 506-530, 1969.

3) Anderson, D. R., Hoyt, W.F. and Hogan, M. J.: The fine structure of the astroglia in the human optic nerve and optic nerve 
head. Trans. Amer. Ophthal. Soc. 65: 275-305, 1967.

4) Bignami, A. and Ralston, H. J. : The cellular reaction to Wallerian degeneration in the central nervous system of the cat. Brain Research 13: 444-461, 1969.

5) Bunge, R.P., Bunge, M. B. and Ris, H. : Electron microscopic study of demyelination in an experimentally induced lesion in adult cat spinal cord. J. Biophys. Biochem. Cytol. $7:$ 685-713, 1960.

6) Cook, R. D.: Observations on glial cells within myelin sheaths in degenerating optic nerve. J. Neurocytol. 3: 737-751, 1974.

7) Cook, R. D. and Wisniewski, H.M.: The role of oligodendroglia and astroglia in Wallerian degeneration of the optic nerve. Brain Research 61:191-206, 1973.

8) Cook, R. D., Ghetti, B. and Wisniewski, H. M.: The pattern of Wallerian degeneration in the optic nerve of newborn kittens : An ultrastructural study. Brain Research 75: 261-275, 1974.

9) Daniel, P. M. and Strich, S. : Histological observations on Wallerian degeneration in the spinal cord of the baboon. Acta Neuropathol. 12: 314-328, 1969.

10) Dunkerly, G. B. and Duncan, D.: A light and electron microscopic study of the normal and degenerating corticospinal tract in the rat. J. Comp. Neurol. 137: 155-184, 1969.

11) Fernando, D. A. : An electron microscopic study of the neuroglial reaction in Wallerian degeneration of the corticospinal tract. Acta Anat. 86: 459-473, 1973.

12) Gonatas, N. K., Levine, S. and Shoulson, R.: Phagocytosis and regeneration of myelin in an experimental leukoencephalopathy. Am. J. Path. 44 : 565-573, 1964.

13) Inoue, Y., Nishimura, Y. and Shimai, K. : On the medullary pyramid and lateral funiculus of the spinal cord of the rhesus monkeys with injured pyramidal tract, surviving for prolonged period. Acta Anat. Nippon. 51 : 349, 1976.

14) Inoue, Y., Nishimura, Y., Inoue, Y., Sugihara, Y., Nakagawa, S. and Shimai, K. : The glioarchitectonics of the chichen optic nerve-Golgi study and electron microscopy-. Okajimas Folia Anat. Jap. 52: 249-272, 1976a.

15) Inoue, $Y .$, Nishimura, $Y .$, Inoue, $Y .$, Sugihara, Y., Nakagawa, S. and Shimai, K.: The glioarchitectonics of the rhesus monkey optic nerve-Electron microscopic study-. Okajimas Folia Anat. Jap. 53 : 45-76, 1976 b.

16) Jacob, A.: Über die feine Histologie der sekundären Faserdegeneration in der weissen Substanz des Rückenmarks (mit besonder Berücksichtigung der Abbauvorgänge). Histol. Histopath. Arb. Nissl Alzheimer 5 : 1-181, 1913.

17) Kusama, T. and Nakazawa, T.: Degeneration and Regeneration in Nervous System-The Basic and Clinical Studies -. Igaku Shoin Ltd. Tokyo, 1975.

18) Lampert, P.W.: Fine structural changes of myelin sheaths in the central nervous system. The structure and Function of Nervous System (Vol. I), pp. 187-204, 1969 (Edited by Boyrne, G. H.).

19) Lampert, P.W. and Cressman, M.R.: Fine-structural changes of myelin sheaths after axonal degeneration in the spinal cord of rats. Am. J. Path. 49 : 1139-1155, 1966.

20) McCaman, R.E. and Robins, E.: Quantitative biochemical studies of Wallerian degeneration in the peripheral and central nervous system-I. J. Neurochem. $5: 18-31,1959$.

21) Mori, S. and Leblond, C.P.: Identification of microglia in light and electron microscopy. J. Comp. Neurol. 135: 5780, 1969a.

22) Mori, S. and Leblond, C. P.: Electron microscopic features and proliferation of astrocytes in the corpus callosum of the rat. J. Comp. Neurol. 137: 197-226, 1969b.

23) Mori, S. and Leblond, C.P.: Electron microscopic identification of three classes of oligodendrocytes and a preliminary study of their proliferative activity in the corpus callosum of young rats. J. Comp. Neurol. 139 : 1-30, 1970.

24) Ressell, G. V.: The compound granular corpuscle or gitter cell: A review, to- 
gether with notes on the origin of this phagocyte. Texas Rept. Biol. Med. 20 : 338-351, 1962.

25) Smith, M.C.: The use of Marchi staining in the later stage of human tract degeneration. J. Neurol. Neurosurg. Psychiat. (N.S.), 14 : 222-225, 1951.

26) Tani, E.: Electron microscopy of secondary degeneration in the central nervous system-Structural changes of axon and myelin sheath -. Advances in
Neurological Sciences 9: 87-101, 1965.

27) Vaughn, J., Hinds, P. L. and Skoff, R. P. : Electron microscopic studies of Wallerian degeneration in rat optic nerves. I. The multipotential glia. J. Comp. Neurol. $140:$ 175-206, 1970.

28) Yamadori, T.: New selective silver impregnation methods of degenerating nerve fibers. Acta Anat. Nippon. 50: 262-266, 1975. 


\section{Explanation of Figures}

\section{Plate I}

Fig. 1. Normal myelinated nerve flbers in a transverse section of the medullary pyramid. Neurofilaments, microtubles, mitochondria (arrows) are clearly revealed. The inset at the lower left shows the neurofilaments which are linked together by bridges of amorphous, electron-dense material (white arrows), and microtubules (a large arrow).

Fig. 2. A degenerated myelinated nerve flber (DF) at 3 days after operation. Filamentous structures are unusually abundant and mitochondria (m) occur in greater in number in the axoplasm in comparison with that of other normal nerve flbers around it. The inset slows unusual filamentous structures. 
Plate I

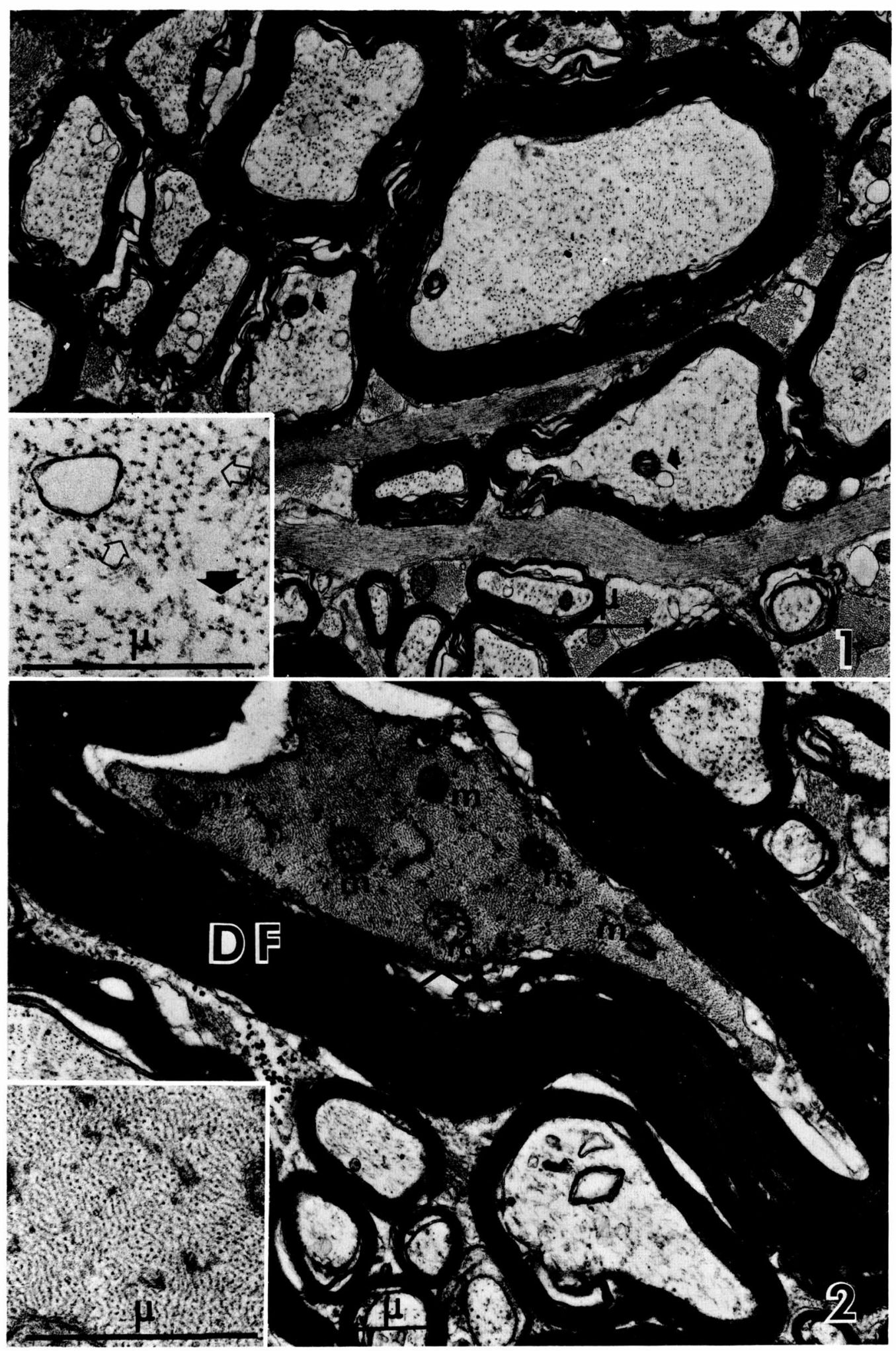




\section{Plate II}

Fig. 3. Various unusual features of the degenerated axon and myelin sheaths at 7 days after operation.

Fig. 3-a. The collapsed myelin sheath. A white arrow shows a part of the process of the phagocyte.

Fig. 3-b. The myelin sheath enclosing a shrunken axon with the dark and finely-granulated axoplasm (a white arrow).

Fig. 3-c. Three concentric rings (shown by three arrow heads) of the myelin sheath of approximately normal thickness.

Fig. 3-d. A unusual myelin mass with a concentric arrangement such that the outer ring was very thick (shown by a large arrow head), and the central ring was of approximately normal thickness (a small arrow head).

Fig. A. The unusual features found in the cross sections of the myelin sheaths at 7 days after operation may be explained by this schema for the invaginating myelin tube. The findings from a to $\mathrm{d}$ in Fig. 3 probably correspond to the features of a cross section through the region $a^{\prime}$ to $d^{\prime}$. 

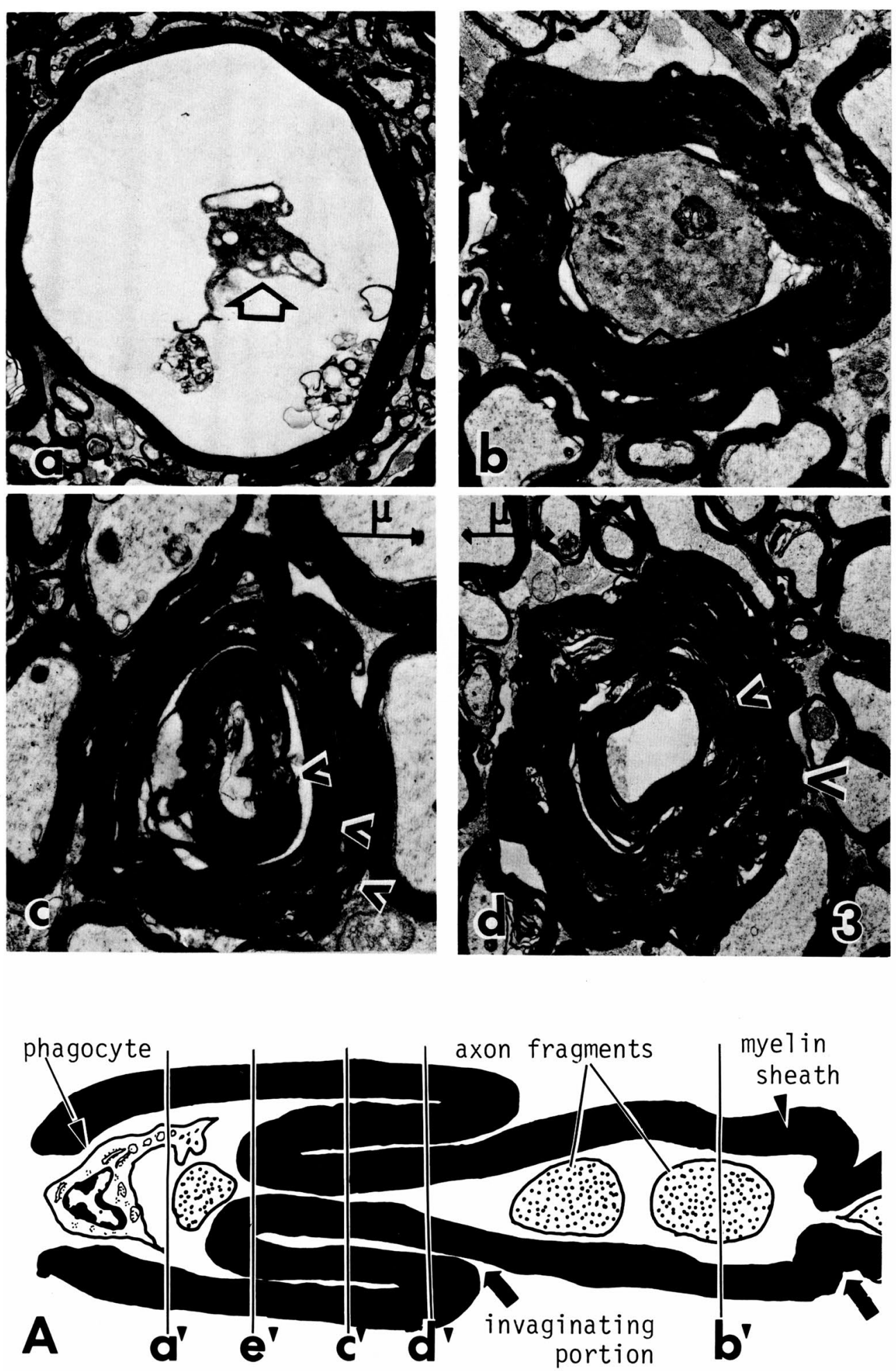


\section{Plate III}

Fig. 4. The phagocyte invading the collapsed myelin tube to engulf the degenerated axon (A). A small arrow head shows small vesicles or short rod-like bodies with the electron-dense material. Vacuoles (V) associated with rough-surfaced endoplasmic reticulum are also observed.

Fig. 5. The phagocyte within the collapsed myelin sheath engulfing the degenerated axon (A) with the light and floccular matrix at 7 days after operation. A black arrow shows the cytoplasm of the phagocyte forming a small pile of thin cytoplasmic sheets around the axon fragment. 


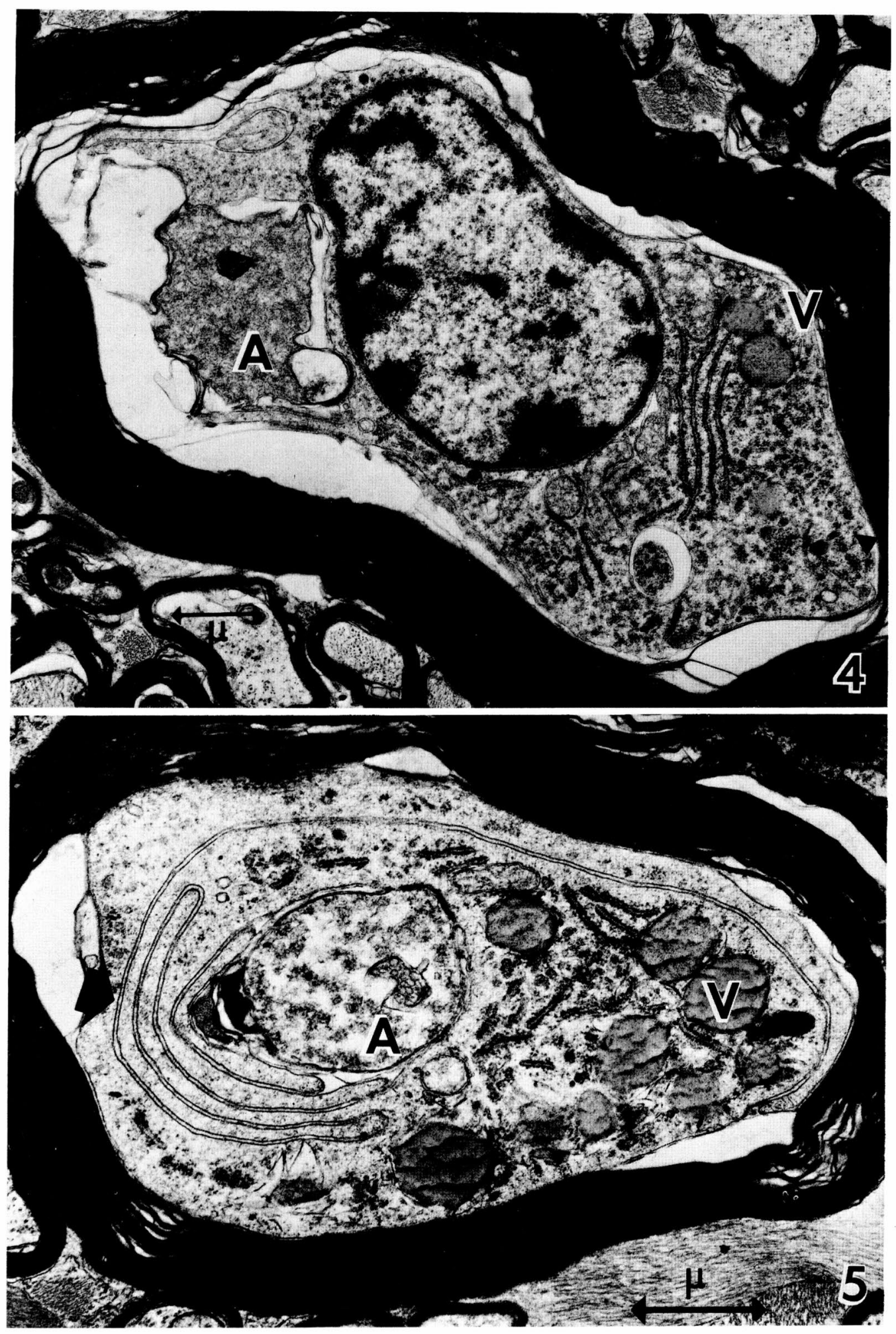




\section{Plate IV}

Fig. 6. The phagocyte within the myelin sheath engulfing the degenerated axon fragment and peeling off the myelin lamellae at 7 days after operation. Two white arrows show the paranodal loops at the node of Ranvier, where the process of the phagocyte invade the myelin lamellae. A black arrow reveals vacuoles with electron-dense material and a small arrow head shows vesicles or short rod-like bodies which were more electron-dense.

Fig. 7. The phagocyte within the lumen of the myelin tube (MT) at 19 days after the first operation (i.e. 12 days after the second operation), engulfing disintegrated myelin fragments $(\mathrm{m})$. Golgi apparatus ( $\mathrm{g}$ ), filaments (f) and numerous empty vacuoles come to be well-developed at this stage. A white arrow shows a thinned myelin sheath enclosing the phagocytes. 

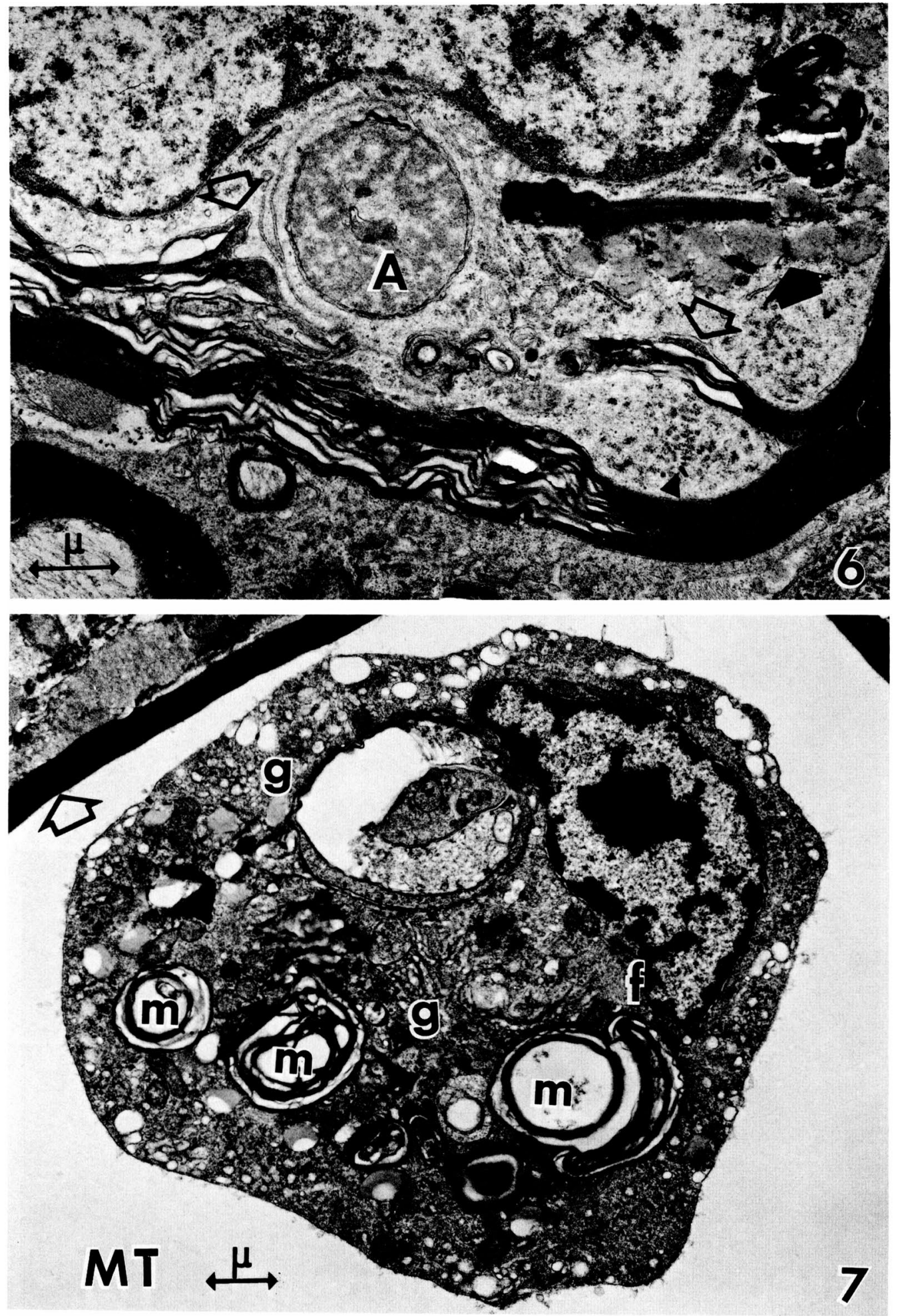


\section{Plate V}

Fig. 8. The phagocyte within the lumen (MT) enclosed by the thinned myelin sheath (an arrow), probably undergoing autolytic breakdown at 19 days after the first operation.

Fig. 9. The phagocyte in the Virchow-Robin space around the blood vessel, surrounding by the basal lamina (an arrow) and connective tissue fibers (ct) at 25 days after operation. The phagocyte contains lipid droplets and crystalline structures which are probably due to the digestion of phagocytosed myelin fragments.

Fig. 10. The phagocyte containing numerous lipid droplets (Lp) and the processes of astrocytes $\left(^{*}\right)$ within the myelin tube $(\mathrm{MT})$ at 33 days after operation. 


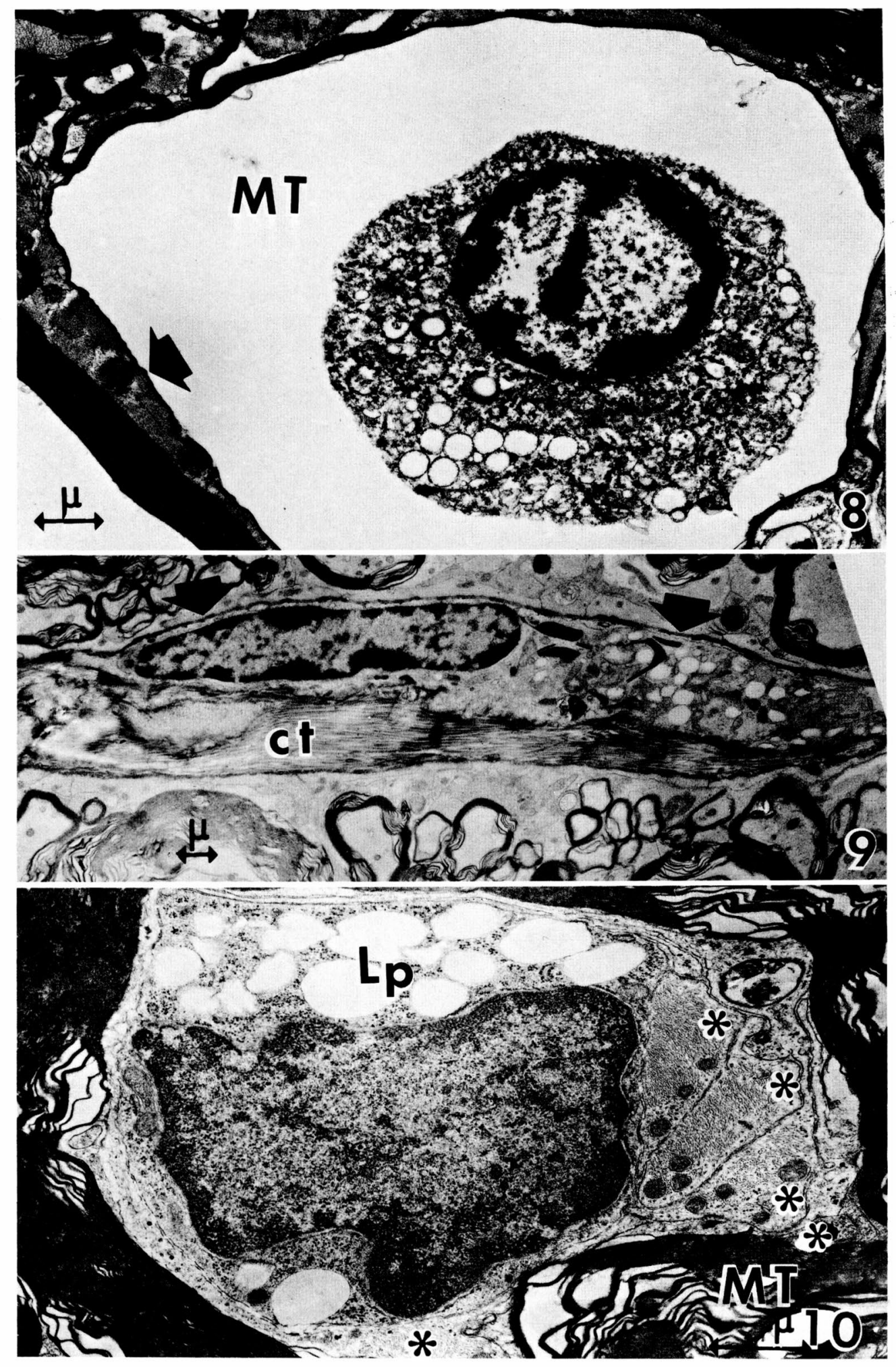




\section{Plate VI}

Fig. 11. The phagocyte occurring among the nerve fibers at 76 days after operation, beginning to engulf myelin fragments $(\mathrm{m})$ again.

Fig. 12. The phagocyte containing numerous lipid droplets and laminated bodies (white arrows) at 117 days after operation, probably due to the digestion of engulfed myelin fragments. Arrow heads shows degenerated thin nerve fibers with the dark axoplasm. 
Plate VI

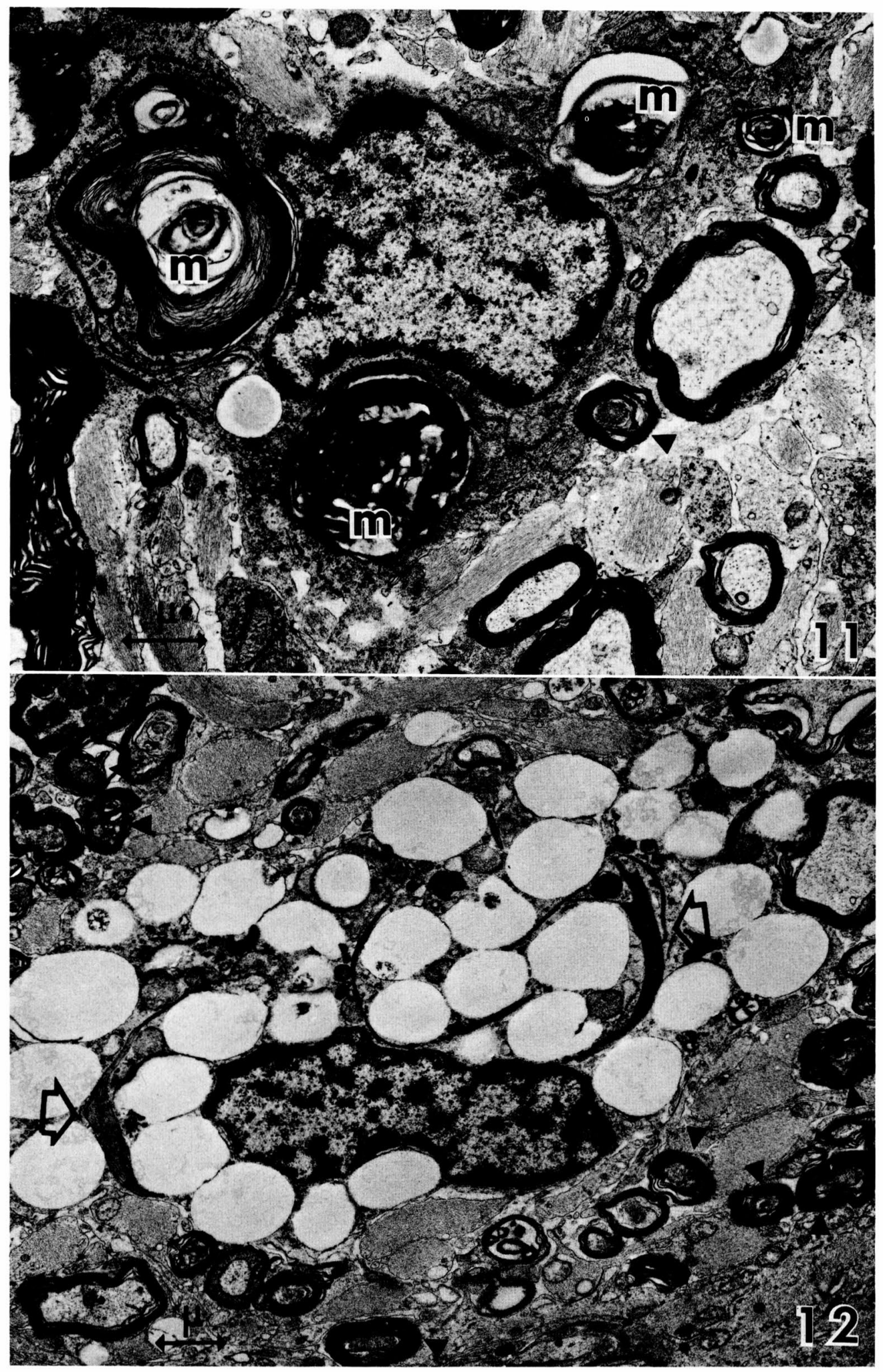




\section{Plate VII}

Fig. 13. Degenerated nerve fibers with the dark axoplasm (arrow heads) in the medullary pyramid at 3 days after operation, and normal nerve fibers (white arrows).

Fig. 14. A degenerated nerve fiber invaded by the phagocyte (a black arrow) at 7 days after operation. An arrow head shows myelin lamellae peeled off by the cytoplasmic tongue of the phagocyte within the myelin sheath. A white arrow shows three concentric rings of the myelin sheath.

Fig. 15. The phagocyte undergoing autolytic breakdown within the thinned myelin tube (an arrow) at 19 days after the first operation (i.e. at 12 days after the second operation).

Fig. 16. The phagocyte containing numerous lipid droplets at 117 days after operation in the medullary pyramid. A white arrow shows thin degenerated fibers distinguishable clearly.

Fig. 17. Degenerated myelin masses (arrow heads) and the phagocyte within myelin sheath (a large arrow) in the lumbar segment of the lateral corticospinal tract at 7 days after operation.

Fig. 18. The phagocytes containing numerous lipid droplets (arrow heads) in the lumbar segment of the lateral corticospinal tract at 117 days after operation. 

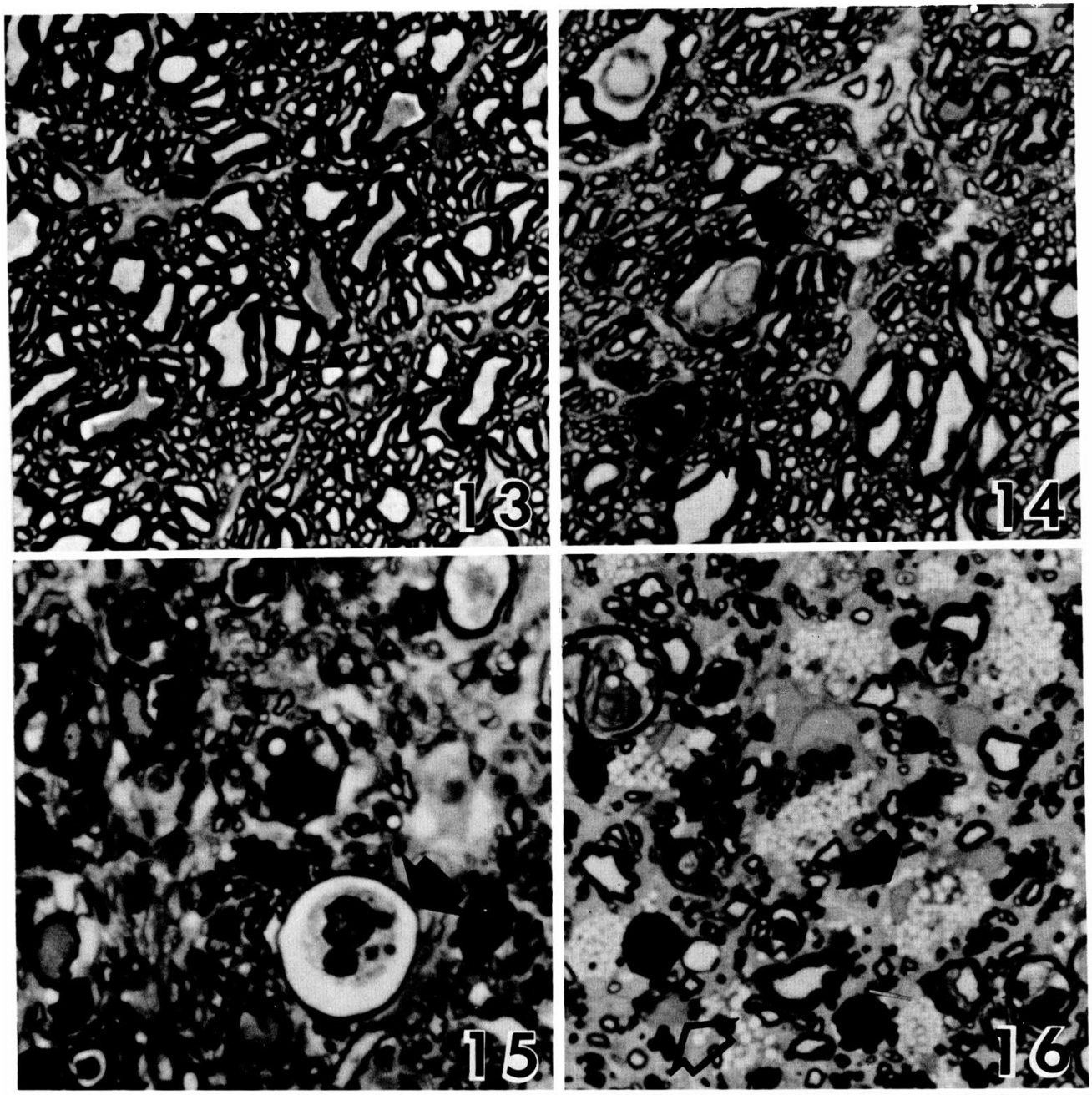

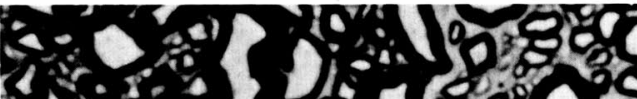

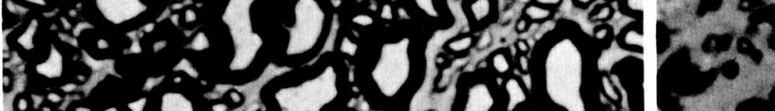

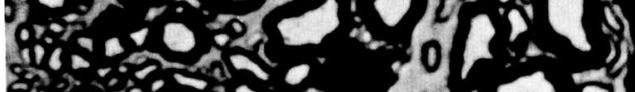

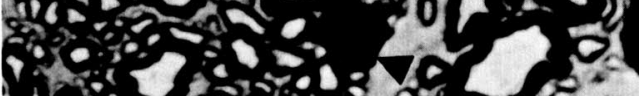

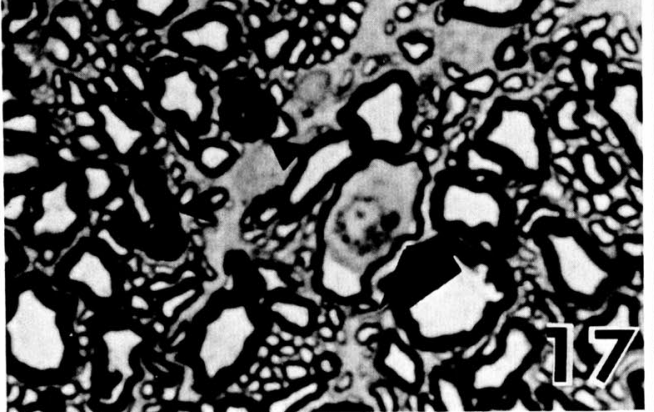

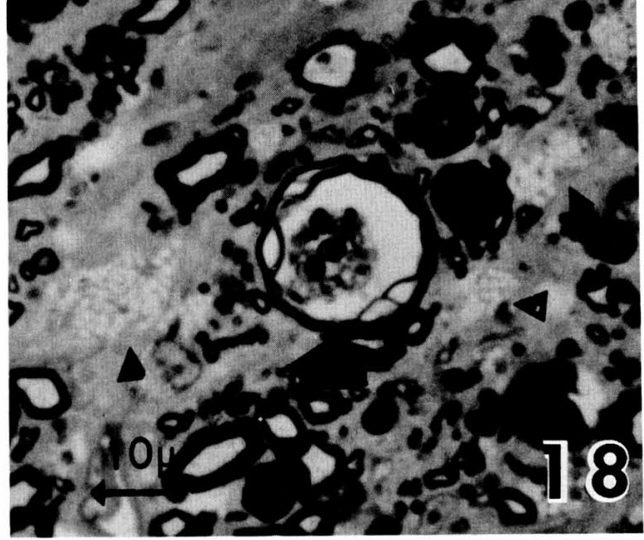

